\title{
Disease Activity and Bone Mineral Density of MCP Joints in Patients with Rheumatoid and Psoriatic Arthritis: Is There a Correlation?-A Study in Patients Treated with Methotrexate and an Anti-TNF $\alpha$ Agent
}

\author{
Ilaria Bertoldi, ${ }^{1}$ Georgios Filippou, ${ }^{1}$ Carlo Alberto Scirè, ${ }^{2}$ \\ Valentina Picerno, ${ }^{1}$ Valentina di Sabatino, ${ }^{1}$ Antonella Adinolfi, ${ }^{1}$ \\ Serena Pierguidi, ${ }^{1}$ Mauro Galeazzi, ${ }^{1}$ and Bruno Frediani ${ }^{1}$ \\ ${ }^{1}$ Department of Medicine, Surgery and Neurosciences-Rheumatology Department, University of Siena, Policlinico le Scotte, \\ Viale Bracci 16, 53100 Siena, Italy \\ ${ }^{2}$ Epidemiology Unit, Italian Society for Rheumatology (SIR), Milan, Italy
}

Correspondence should be addressed to Ilaria Bertoldi; bertoldiilaria@libero.it

Received 22 September 2013; Accepted 30 October 2013

Academic Editors: W. H. Abdulahad, M. Benucci, R. Marks, and S. Ozgocmen

Copyright (C) 2013 Ilaria Bertoldi et al. This is an open access article distributed under the Creative Commons Attribution License, which permits unrestricted use, distribution, and reproduction in any medium, provided the original work is properly cited.

Background. Bone damage in rheumatoid arthritis (RA) and in psoriatic arthritis (PsA) includes an accelerated bone mineral density (BMD) reduction. The objective was to evaluate BMD variations of the metacarpophalangeal joints (MCPs) in patients starting treatment with methotrexate (MTX) or etanercept. Methods. Patients affected by RA or PsA with hand joints involvement and with moderate or high disease activity, were enrolled in this study. All patients underwent clinical examination, laboratory exams, and a DXA scan of the most affected hand, as assessed with an ultrasound examination at the baseline, at the time of enrolment and after 1, 3, 6, and 12 months. Patients non-responders to MTX received combination therapy, while patients with no previous treatment initiated MTX. Results. 22 patients were enrolled. In both RA and PsA groups, BMD increased independently of the treatment. However, in the patients affected by RA, a slight BMD decrease was observed at the last checkup. Globally, the BMD variations of the MCPs were strongly correlated with the disease activity. At the reduction of DAS28, the scores corresponded an increase of BMD. Conclusions. MCPs BMD is inversely correlated to disease activity. BMD increase seems to be correlated with the response to treatment and not with the drug itself.

\section{Introduction}

Bone damage in rheumatoid arthritis (RA) and in psoriatic arthritis (PsA) includes joint damage and accelerated bone mineral density (BMD) reduction [1], both at a local and generalised level. Bone damage is caused by an increased osteoclast activity and decreased osteoblast activation. This is mostly mediated by tumor necrosis factor (TNF)- $\alpha$, interleukin (IL)-1, IL-6, IL-17, and receptor activator of nuclear factor kappa B ligand (RANKL) [2-5]. The erosion represents the final result of this process $[6,7]$ and it can be considered the central feature of bone damage of both RA and PsA, although in PsA there are significant differences when compared with RA, with a pattern characterised by concurrent erosions and new bone formation [8-10]. However, bone in the proximity of inflamed joints is susceptible to $\mathrm{BMD}$ reduction and it precedes erosive damage on X-ray [11-16].

Dual energy X-ray absorptiometry (DEXA, DXA) is the gold standard for measuring BMD [17]. Previous clinical studies demonstrated an association between hand BMD and RA severity, including disease activity, functional impairment and joint destruction [11-16, 18-22]. However, only a little data is available on the association between hand BMD reduction and disease activity in patients who are treated 


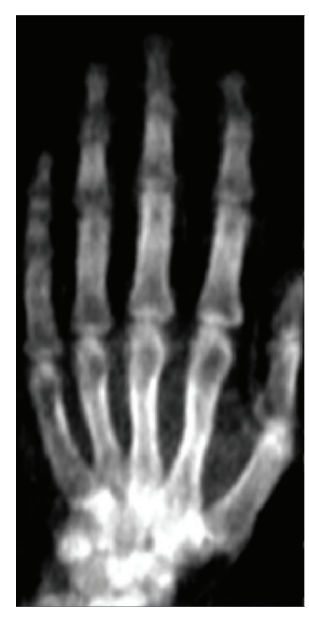

(a)

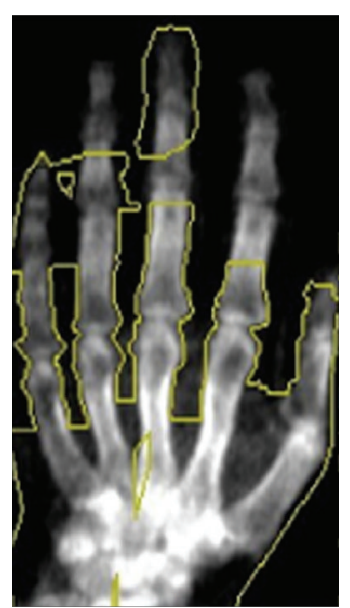

(b)

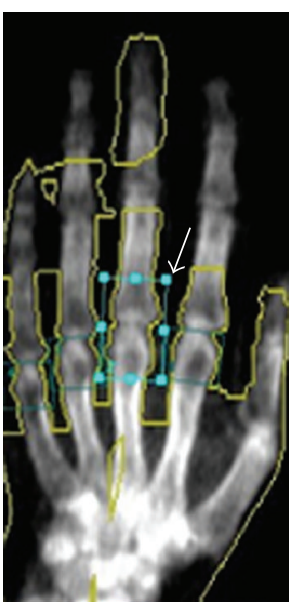

(c)

Figure 1: Acquisition and analysis of the MCP BMD at the first visit. The machine acquires the hand region (a) that has to be analysed manually. Then the operator defines the borders of the bone working in a magnified image with the software of the densitometer, obtaining a mask visible in the second image (white line) (b). Then he creates a ROI (region of interest, arrow) that includes the MCP rim, the head of the metacarpal bone, and the basis of the proximal phalange (c). Both the mask and the ROI are then saved and always used to assess BMD changes of each patient.

with disease modifying anti-rheumatic drugs (DMARDs) and TNF $\alpha$ inhibitors in a tight controlled setting [23-26].

The aim of this longitudinal observational study was to examine the BMD variations of the metacarpophalangeal joints (MCP) in patients affected by RA or PsA, in treatment with a DMARD-methotrexate-(MTX) or an anti-TNF $\alpha$ agent-etanercept.

\section{Patients and Methods}

All consecutive patients that attended our outpatients clinic for a six-month period (June-December 2011), affected by RA or PsA with hand joints involvement and with moderate or high disease activity as defined by the DAS28 (>3.2), were enrolled in this study. An RA diagnosis was made according to the previous ACR criteria [27], while a PsA diagnosis was made according to the Caspar criteria [28]. Patients could be either at the first diagnosis of disease or already in treatment with DMARDs but with a moderate-high disease activity as defined by DAS28 values, and they were eligible for antiTNF $\alpha$ administration as defined by the Italian guidelines $[29,30]$. Other inclusion criteria were an age of more than 18 years and a stable dose of steroids and methotrexate (for the second group) for the last 3 months. Exclusion criteria were pregnancy, other concomitant treatments that could influence BMD, malignancies, infectious diseases, chronic heart failure class III-IV according to the New York Heart Association (NYHA), severe pulmonary and hepatic diseases, unstable dosage of steroids or steroid doses superior of $10 \mathrm{mg}$ of prednisone (or equivalent) for the second group of patients, or parenteral administration of steroids prior to the enrollment. A high dosage of steroids with quick tapering was allowed for the group at the first diagnosis, if administered for the first time. Nonsteroid anti-inflammatory drugs (NSAIDs) and local steroid injections in joints other than hands were permitted during the study. All patients agreed to participate in the study and signed an informed consent.

All patients underwent a clinical examination (all parameters necessary for the DAS28-CRP calculation) at the time of enrollment (time 0 , T0) and after 1 month (T1), 3 months (T2), 6 months (T3), and 12 months (T4). At the time of enrollment, all patients also underwent an US examination of the MCP of both hands in order to assess the most "active" joint. All MCP were examined according to the EULAR recommendations [31], while inflammation was assessed using a semiquantitative score for synovial proliferation and power Doppler signal in a $0-3$ scale as described previously [32]. The most active joint was the joint that reached the higher score for synovial proliferation plus a power Doppler signal. Joint effusion was not taken into account for this evaluation. Clinical examination and ultrasonography were performed by independent operators, blind to each others findings.

DXA examination of the hand, for the BMD assessment, was performed at T0, T2, T3, and T4. Joint BMD was measured at the most active joint, as defined at the US examination, with a dedicated region of interest (ROI) created ad hoc for the joint. Then the tool "compare mask" was used for the evaluation of the joint during the study in order to ensure the maximum reliability. In fact, the "compare mask" tool superimposes the images acquired during the followup and allows a very similar positioning of the ROI in the joint of interest (Figure 1). A Lunar Prodigy machine with the enCORE software was used for the study; the quality assurance data were collected daily to guaranty the performance of the scanners. The coefficient of variation (CV) of the machine used for the study has been previously tested for other sites and was never superior to 1.6\% (lumbar spine $1.1 \%$, femoral neck 1.5\%, total femur 1.6\%) [33]. Using 


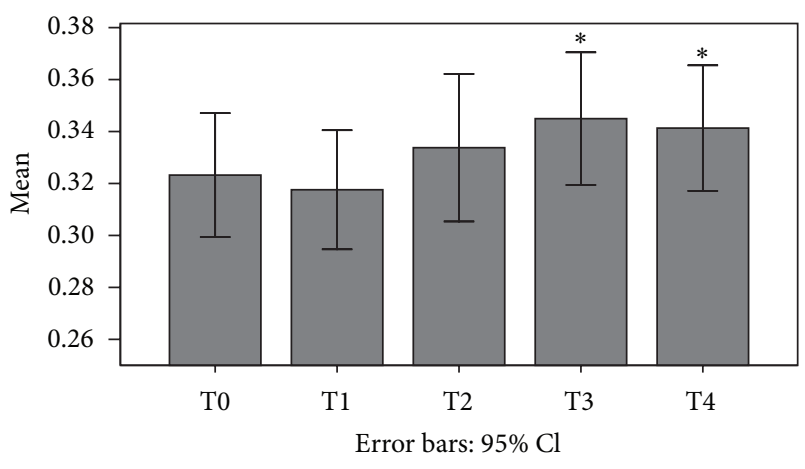

FIGURE 2: BMD variations of our cohort during the follow-up period. $\left({ }^{*} P<0.01\right)$.

the same machine, with a similar study design to ours (dedicated ROIs on MCP joints), Naumann et al. found a CV from $1.23 \%$ to $2.48 \%$ for MCP (MCP II-V: mean CV $1.16 \%$; mean Least Significant Change 3.25\%) [34].

Descriptive measures of demographic, disease-related, and DXA variables are presented as absolute and relative frequencies, mean and standard deviation (SD), or median and interquartile range (IQR) based on their type and distribution. The correlation between the variables has been calculated using multiple regression analysis as proposed by Bland and Altman [35]. Partial correlation coefficient between $\triangle \mathrm{BMD}$ (beginning and end of the study) and the area under the curve of DAS28 over the study period has been used for the assessment of association between disease activity and BMD. Analyses were performed using STATA software package (StataCorp, 2009, release 11, TX, USA).

\section{Results}

Twenty-two patients ( 7 male, mean age of 49.9 years old, SD 12.4) were enrolled in the study. Of these patients 10 were affected by PsA and 12 by RA. Twelve patients were at the first diagnosis (early disease, $7 \mathrm{RA}$ ) and were treated with MTX and 10 patients (5 RA) were nonresponders to DMARDs and were treated with MTX plus an anti-TNF $\alpha$ agent (etanercept). The mean (SD) disease duration of the nonresponders was 29.9 (9.7) months. Demographic characteristics and baseline clinical data of the patients are summarised in Table 1.

Globally, the mean BMD values increased during the follow-up period (Figure 2), in a statistically significant way, and also DAS28 improved. However, the BMD did not increase equally in all patient groups and in all treatment groups.

Patients affected by PsA presented with a higher BMD at the beginning of the study, despite the disease duration. In fact both new patients and nonresponders to MTX had a higher BMD (Figure 3). During the first month we assisted in a decrease of the BMD of the affected joint in all groups of patients. At the end of the study period patients in treatment with etanercept maintained the BMD increases obtained while patients in treatment with MTX had a slight reduction

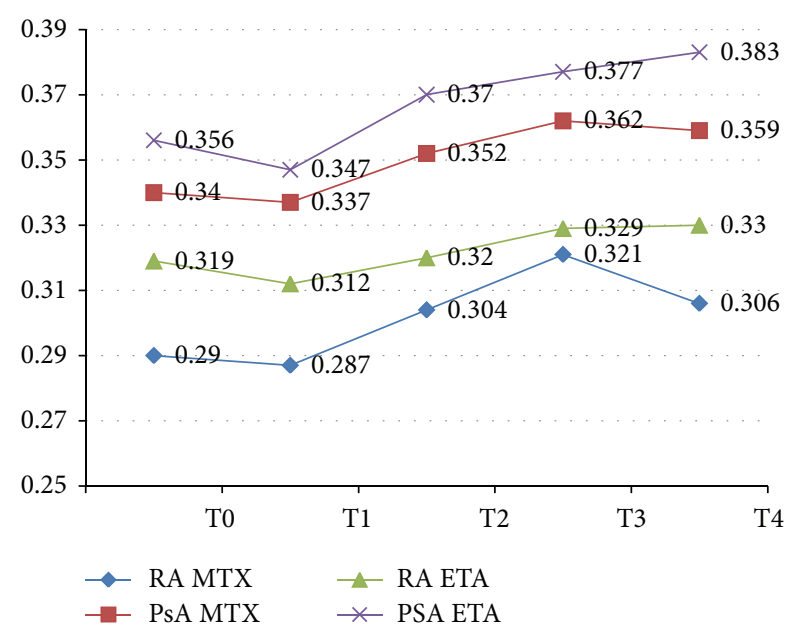

FIgURE 3: BMD values of the patients in the RA group and PsA group.

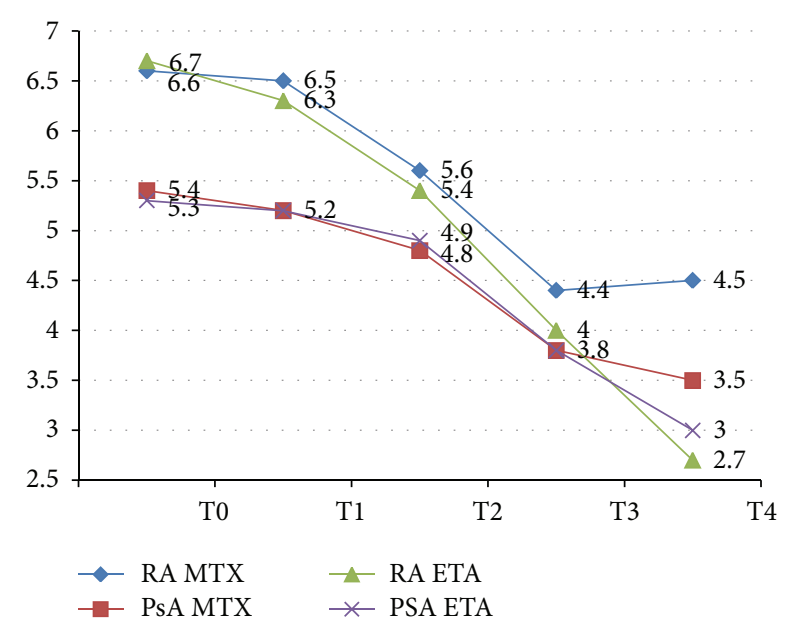

FIGURE 4: DAS28 values of the patients in the different groups during the study time.

of the BMD at the last checkup, that resulted statistically significant only at the RA group $(P<0.05$ versus T3).

DAS28 also improved in all patient groups. However in the MTX group we observed a higher score and not all patients reached a low disease activity with MTX alone (Figure 4).

Multiple regression analysis [35] was used to assess the correlation between disease activity and BMD of the MCP joints. The correlation coefficient is -0.56 with $P<0.0001$. That means that the variation of DAS28 is associated with an inverse variation of the BMD of MCP joints. In other words, a decrease of the disease activity is associated with an increase of MCPs BMD and vice versa.

Further, the exposure at high disease activity scores is correlated with low values of MCPs' BMD independent of the sex or age of the patients (partial correlation coefficient $-0.493, P=0.028)$. 
TABle 1: Demographic and clinical data of the patients at baseline.

\begin{tabular}{|c|c|c|c|c|}
\hline & \multicolumn{2}{|c|}{ Patients with RA } & \multicolumn{2}{|c|}{ Patients with PsA } \\
\hline & MTX group & Etanercept group & MTX group & Etanercept group \\
\hline Patient, $N$ (females) & $7(5)$ & $5(4)$ & $5(2)$ & $5(1)$ \\
\hline Age, mean (SD) & $45.7(11.7)$ & $60.0(10)$ & $45.4(14.1)$ & $50.2(11)$ \\
\hline Disease duration (months), mean (SD) & $1.7(0.2)$ & $26.8(9.5)$ & $1.8(0.3)$ & $33(9.5)$ \\
\hline DAS28, mean (SD) & $6.6(0.9)$ & $6.7(0.9)$ & $5.4(0.7)$ & $5.3(0.6)$ \\
\hline BMD of the examined joint, mean (SD) & $0.291(0.067)$ & $0.319(0.028)$ & $0.340(0.018)$ & $0.363(0.057)$ \\
\hline
\end{tabular}

PDUS: power Doppler US. DAS values between groups were not statistically significant.

At 12 months, 5 patients were classified as "nonresponder" (3 affected by RA, in treatment with methotrexate, 2 affected by PsA, one in treatment with methotrexate and one in treatment with etanercept).

\section{Discussion}

Periarticular osteoporosis in patients affected by arthritis is a well-known phenomenon [1-5]. In fact, before the introduction of more sophisticated imaging techniques for the diagnosis of arthritis and joint effusion, the finding of MCPs transparency in traditional X-rays was one of the items of the 1987 ACR classification criteria for Rheumatoid Arthritis [27]. More recent studies have also demonstrated that periarticular bone loss occurs also in MCPs of patients affected by Psoriatic Arthritis [16, 23, 24] even if this aspect has been debated. In fact, periarticular osteoporosis is not considered by all authors as a marker of disease in the case of PsA, interpreting plain radiographs $[8,23,24]$. Using a BMD measurement, Reid et al. reported that BMD in 12 patients with PsA (assessed by metacarpal index) was not different from controls [36]. Whereas Cooper et al., using single photon absorptiometry (SPA) measurements of the distal forearm, noted bone loss in both RA and PsA, suggesting that their patients had more active disease than those of Reid et al. [37]. One previous study of our group assessed periarticular BMD in patients with various inflammatory arthropathies, and it found that periarticular bone loss occurred even in patients with PsA [38].

The pathogenesis of periarticular osteopenia in both rheumatoid and psoriatic arthritis is related to osteoclast activation by proinflammatory cytokines from the inflamed synovium, including prostaglandin E2, osteoclast activating factor, tumour necrosis factor (TNF), and interleukin 1 [15]. The ability of anti-inflammatory treatment to reduce hand bone loss in RA has been demonstrated in a double-blind study comparing oral prednisolone $7.5 \mathrm{mg}$ /day for 2 years with placebo. The prednisolone group had less hand BMD loss, suggesting that the potent anti-inflammatory effect of prednisolone exceeded its negative effect on the bone [39].

With respect to the effects of anti-TNF therapy on hand bone loss in RA, only a few studies have been conducted. RA patients treated with anti-TNF therapy have been shown to have a lower rate of bone loss at the spine and hip than at the hand $[18,24-26]$. In a 2-year longitudinal treatment strategy study (the BeST study), RA patients treated with antiTNF therapy or high-dose prednisolone were shown to have a lower rate of bone loss at the hand than patients treated with conventional DMARD [18]. Furthermore, in a study employing quantitative ultrasound, the use of anti-TNF therapy had a positive effect on periarticular bone [40]. The beneficial effect of anti-TNF treatment on inflammatory-related hand bone loss in RA is supported by analysis from the PREMIER study, in which hand bone loss was assessed by digital Xray radiogrammetry on the same hand radiographs scored with modified Sharp score [25]. In this study, the authors concluded that in patients with RA, potent anti-TNF therapy reduces the rate of inflammatory-related hand bone. This study also suggests that the bone damage disease process is still present in RA patients treated with TNF antagonists, even if radiographic joint damage on radiographs is apparently arrested and they suggest that quantitative measures of hand bone loss in RA patients can be recommended as outcomes for future clinical trials to detect ongoing bone damage.

In our series of patients, periarticular BMD increased after the third month of followup in both groups of patients except the last 3 months where we observed a negative trend in the group of patients affected with RA and treated with MTX. This could be due to the presence of 3 nonresponders in this group. This is in accordance with the previous observations on the effects of an appropriate anti-inflammatory therapy on bone loss in patients with RA [25, 39, 40]. In addition, we observed that BMD also increases in patients affected by PsA, independent of the treatment strategy. This observation is in accordance with the results of the study by Hoff et al. [23]. However, this is partially in contrast with the results of Szentpetery et al. [24]. In their study, they observed that there is a slight reduction of the periarticular BMD in MCPs either in patients affected by RA or PsA during the first year of treatment with an anti-TNF $\alpha$ agent. This is rather surprising and the same authors admitted that this result was rather unexpected.

In this study, the BMD variations have demonstrated to be inversely proportional to the disease activity defined by DAS28 scores. In previous studies, a good response to the treatment was correlated with reduced bone loss in patients affected by RA [25] and by PsA [23]. However, this is the first study where a BMD gain has been observed and not only with a reduction of the rate of bone loss. This could be due to the methods of the study. In our study we assessed only the most inflamed MCP joint according to clinical and US findings, so it is reasonable to expect a more dramatic response to the treatment either from the inflammation or from the bone damage point of view. In the other 2 studies, digital 
radiogrammetry (DXR) has been applied in more MCPs independently of the grade of inflammation of those joints. This could explain the less dramatic variation of BMD in these two studies. Also, in our study we discovered that there is a statistically significant correlation between persistence of disease activity and MCP BMD, independent of the age and sex of the patient.

Our study has some limitations that should be discussed. First of all the number of patients stratified by diagnosis, age, sex, and therapy was not sufficient to allow a statistical comparison between the various treatment groups, other than the descriptive ones. These analyses allowed us to observe that patients with a new diagnosis of RA tended to have a lower MCP BMD than patients already treated with a DMARD. Also PsA patients tended to have a higher BMD in respect to those affected by RA independent of the treatment administered. This is in accordance with previous observations [16]. Also, patients in combination therapy etanercept plus MTX, independent of the diagnosis, presented lower DAS28 scores and tended to obtain higher BMD values at the end of the followup in respect to the patients in monotherapy.

The assessment of only one, the most inflamed, MCP could be considered another limitation of our study. However, we chose to assess only one joint as in previous studies, the evaluation of more MCPs demonstrated a reduced rate or an arrest of bone loss $[23,25]$ while in our case we observed in some patients a gain of BMD at the end of the study period. It is reasonable to believe that the most inflamed joint reflects the bone damage caused by inflammation better. For example, in the study by Deodhar et al. [22], the authors assessed the bone mineral content of the whole hand and even if they also observed a reduced bone loss rate in the patients treated, the end of the study (5 years) resulted a consistent reduction of the BMC, especially during the first 3 years. We believe that the treatment options (only traditional DMARDs), the long study period, and maybe the inclusion of joints that are not typically affected by RA as well as the diaphysis of the hand's bones could have influenced the final results, adding the systemic bone damage (systemic osteoporosis) to bone damage due to local inflammation.

In conclusion, in patients affected by RA or PsA an effective treatment with either traditional DMARDs or anti$\mathrm{TNF} \alpha$ agents is effective in reducing periarticular bone loss in active MCPs. Bone loss reduction seems to be related to the treatment efficacy at a systemic level (reduction of DAS28 scores) more than the treatment agent itself even if patients treated with combination therapy tend to recuperate higher values of MCP BMD than those in monotherapy both in RA and PsA. Finally, further studies could be useful for assessing the role of the DXA measurements of affected MCPs and for defining the correlation between bone loss and erosive damage in RA and PsA.

\section{Acknowledgments}

The authors would like to express their appreciation to Dr. Greta Carrara for her contribution in the statistical analysis.

\section{References}

[1] E. M. Gravallese, "Bone destruction in arthritis," Annals of the Rheumatic Diseases, vol. 61, supplement 2, pp. ii84-ii86, 2002.

[2] T. Hirayama, L. Danks, A. Sabokbar, and N. A. Athanasou, "Osteoclast formation and activity in the pathogenesis of osteoporosis in rheumatoid arthritis," Rheumatology, vol. 41, no. 11, pp. 1232-1239, 2002.

[3] N. C. Walsh, T. N. Crotti, S. R. Goldring, and E. M. Gravallese, "Rheumatic diseases: the effects of inflammation on bone," Immunological Reviews, vol. 208, pp. 228-251, 2005.

[4] G. Schett, "Review: immune cells and mediators of inflammatory arthritis," Autoimmunity, vol. 41, no. 3, pp. 224-229, 2008.

[5] S. Herman, G. Krönke, and G. Schett, "Molecular mechanisms of inflammatory bone damage: emerging targets for therapy," Trends in Molecular Medicine, vol. 14, no. 6, pp. 245-253, 2008.

[6] S. R. Goldring, "Pathogenesis of bone erosions in rheumatoid arthritis," Current Opinion in Rheumatology, vol. 14, no. 4, pp. 406-410, 2002.

[7] D. O’Gradaigh, D. Ireland, S. Bord, and J. E. Compston, "Joint erosion in rheumatoid arthritis: interactions between tumour necrosis factor $\alpha$, interleukin 1 , and receptor activator of nuclear factor $\kappa \mathrm{B}$ ligand (RANKL) regulate osteoclasts," Annals of the Rheumatic Diseases, vol. 63, no. 4, pp. 354-359, 2004.

[8] V. Wright, "Psoriatic arthritis: a comparative radiographic study of rheumatoid arthritis and arthritis associated with psoriasis," Annals of the Rheumatic Diseases, vol. 20, pp. 123-132, 1961.

[9] S. Finzel, M. Englbrecht, K. Engelke, C. Stach, and G. Schett, "A comparative study of periarticular bone lesions in rheumatoid arthritis and psoriatic arthritis," Annals of the Rheumatic Diseases, vol. 70, no. 1, pp. 122-127, 2011.

[10] S. R. Goldring and M. B. Goldring, "Eating bone or adding it: the Wnt pathway decides," Nature Medicine, vol. 13, no. 2, pp. 133-134, 2007.

[11] M. Güler-Yüksel, N. B. Klarenbeek, Y. P. M. GoekoopRuiterman et al., "Accelerated hand bone mineral density loss is associated with progressive joint damage in hands and feet in recent-onset rheumatoid arthritis," Arthritis Research and Therapy, vol. 12, no. 3, p. R96, 2010.

[12] M. Hoff, G. Haugeberg, and T. K. Kvien, "Hand bone loss as an outcome measure in established rheumatoid arthritis: 2-year observational study comparing cortical and total bone loss," Arthritis Research and Therapy, vol. 9, no. 4, article R81, 2007.

[13] T. Jensen, M. Hansen, K. E. Jensen, J. Pødenphant, T. M. Hansen, and L. Hyldstrup, "Comparison of dual X-ray absorptiometry (DXA), digital X-ray radiogrammetry (DXR), and conventional radiographs in the evaluation of osteoporosis and bone erosions in patients with rheumatoid arthritis," Scandinavian Journal of Rheumatology, vol. 34, no. 1, pp. 27-33, 2005.

[14] G. Hougeberg, M. C. Lodder, W. F. Lems et al., "Hand cortical bone mass and its associations with radiographic joint damage and fractures in 50-70 year old female patients with rheumatoid arthritis: cross sectional Oslo-Truro-Amsterdam (OSTRA) collaborative study," Annals of the Rheumatic Diseases, vol. 63, no. 10, pp. 1331-1334, 2004.

[15] T. Jensen, M. Klarlund, M. Hansen et al., "Bone loss in unclassified polyarthritis and early rheumatoid arthritis is better detected by digital $\mathrm{x}$ ray radiogrammetry than dual $\mathrm{x}$ ray absorptiometry: relationship with disease activity and radiographic outcome," Annals of the Rheumatic Diseases, vol. 63, no. 1, pp. 15-22, 2004. 
[16] B. J. Harrison, C. E. Hutchinson, J. Adams, I. N. Bruce, and A. L. Herrick, "Assessing periarticular bone mineral density in patients with early psoriatic arthritis or rheumatoid arthritis," Annals of the Rheumatic Diseases, vol. 61, no. 11, pp. 1007-1011, 2002.

[17] W. Sturtridge, B. Lentle, and D. A. Hanley, "Prevention and management of osteoporosis: consensus statements from the Scientific Advisory Board of the Osteoporosis Society of Canada. 2. The use of bone density measurement in the diagnosis and management of osteoporosis," Canadian Medical Association Journal, vol. 155, no. 7, pp. 924-929, 1996.

[18] M. Güler-Yüksel, C. F. Allaart, Y. P. M. Goekoop-Ruiterman et al., "Changes in hand and generalised bone mineral density in patients with recent-onset rheumatoid arthritis," Annals of the Rheumatic Diseases, vol. 68, no. 3, pp. 330-336, 2009.

[19] G. Haugeberg, M. J. Green, M. A. Quinn et al., "Hand bone loss in early undifferentiated arthritis: Evaluating bone mineral density loss before the development of rheumatoid arthritis," Annals of the Rheumatic Diseases, vol. 65, no. 6, pp. 736-740, 2006.

[20] J. Böttcher, A. Pfeil, A. Rosholm et al., "Computerized quantification of joint space narrowing and periarticular demineralization in patients with rheumatoid arthritis based on digital x-ray radiogrammetry," Investigative Radiology, vol. 41, no. 1, pp. 3644, 2006.

[21] W. B. Jawaid, D. Crosbie, J. Shotton, D. M. Reid, and A. Stewart, "Use of digital $\mathrm{x}$ ray radiogrammetry in the assessment of joint damage in rheumatoid arthritis," Annals of the Rheumatic Diseases, vol. 65, no. 4, pp. 459-464, 2006.

[22] A. A. Deodhar, J. Brabyn, I. Pande, D. L. Scott, and A. D. Woolf, "Hand bone densitometry in rheumatoid arthritis, a five year longitudinal study: an outcome measure and a prognostic marker," Annals of the Rheumatic Diseases, vol. 62, no. 8, pp. 767-770, 2003.

[23] M. Hoff, A. Kavanaugh, and G. Haugeberg, "Hand bone loss in patients with psoriatic arthritis: posthoc analysis of IMPACT II data comparing infliximab and lacebo," The Journal of Rheumatology, vol. 40, no. 8, pp. 1344-1348, 2013.

[24] A. Szentpetery, M. J. McKenna, B. F. Murray et al., "Periarticular bone gain at proximal interphalangeal joints and changes in bone turnover markers in response to tumor necrosis factor inhibitors in rheumatoid and psoriatic arthritis," The Journal of Rheumatology, vol. 40, no. 5, pp. 653-662, 2013.

[25] M. Hoff, T. K. Kvien, J. Kälvesten, A. Elden, A. Kavanaugh, and G. Haugeberg, "Adalimumab reduces hand bone loss in rheumatoid arthritis independent of clinical response: subanalysis of the PREMIER study," BMC Musculoskeletal Disorders, vol. 12, article 54, 2011.

[26] M. Vis, E. A. Havaardsholm, G. Haugeberg et al., "Evaluation of bone mineral density, bone metabolism, osteoprotegerin and receptor activator of the $\mathrm{NF} \kappa \mathrm{B}$ ligand serum levels during treatment with infliximab in patients with rheumatoid arthritis," Annals of the Rheumatic Diseases, vol. 65, no. 11, pp. 1495-1499, 2006.

[27] F. C. Arnett, S. M. Edworthy, D. A. Bloch et al., "The American Rheumatism Association 1987 revised criteria for the classification of rheumatoid arthritis," Arthritis and Rheumatism, vol. 31, no. 3, pp. 315-324, 1988.

[28] W. Taylor, D. Gladman, P. Helliwell, A. Marchesoni, P. Mease, and H. Mielants, "Classification criteria for psoriatic arthritis: development of new criteria from a large international study," Arthritis and Rheumatism, vol. 54, no. 8, pp. 2665-2673, 2006.
[29] R. Caporali, F. Conti, S. Alivernini et al., "Recommendations for the use of biologic therapy in rheumatoid arthritis: update from the Italian Society for Rheumatology I. Efficacy," Clinical and Experimental Rheumatology, vol. 29, no. 3, supplement 66, pp. S7-S14, 2011.

[30] C. Salvarani, N. Pipitone, A. Marchesoni et al., "Recommendations for the use of biologic therapy in the treatment of psoriatic arthritis: update from the Italian Society for Rheumatology," Clinical and Experimental Rheumatology, vol. 29, no. 3, supplement 66, pp. S28-S41, 2011.

[31] M. Backhaus, G.-R. Burmester, T. Gerber et al., "Guidelines for musculoskeletal ultrasound in rheumatology," Annals of the Rheumatic Diseases, vol. 60, no. 7, pp. 641-649, 2001.

[32] A. K. Scheel, K.-G. A. Hermann, E. Kahler et al., "A novel ultrasonographic synovitis scoring system suitable for analyzing finger joint inflammation in rheumatoid arthritis," Arthritis and Rheumatism, vol. 52, no. 3, pp. 733-743, 2005.

[33] B. Frediani, "Effects of two administration schemes of intramuscular clodronic acid on bone mineral density: a randomized, open-label, parallel-group study," Clinical Drug Investigation, vol. 31, no. 1, pp. 43-50, 2011.

[34] L. Naumann, K.-G. A. Hermann, D. Huscher et al., "Quantification of periarticular demineralization and synovialitis of the hand in rheumatoid arthritis patients," Osteoporosis International, vol. 23, no. 12, pp. 2671-2679, 2012.

[35] J. M. Bland and D. G. Altman, "Calculating correlation coefficients with repeated observations: part I-correlation within subjects," British Medical Journal, vol. 310, no. 6977, article 446, 1995.

[36] D. M. Reid, N. S. J. Kennedy, J. Nicoll, M. A. Smith, P. Tothill, and G. Nuki, "Total and peripheral bone mass in patients with psoriatic arthritis and rheumatoid arthritis," Clinical Rheumatology, vol. 5, no. 3, pp. 372-378, 1986.

[37] C. Cooper, V. Poll, M. McLaren, S. O. Daunt, and M. I. D. Cawley, "Alterations in appendicular skeletal mass in patients with rheumatoid, psoriatic, and osteoarthropathy," Annals of the Rheumatic Diseases, vol. 47, no. 6, pp. 481-484, 1988.

[38] B. Frediani, A. Allegri, P. Falsetti et al., "Juxta-articular osteoporosis in arthritis and arthrosis: fan-beam $\mathrm{x}$ ray densitometry," Arthritis and Rheumatism, vol. 42, supplement, p. S355, 1999.

[39] G. Haugeberg, A. Strand, T. K. Kvien, and J. R. Kirwan, "Reduced loss of hand bone density with prednisolone in early rheumatoid arthritis: results from a randomized placebocontrolled trial," Archives of Internal Medicine, vol. 165, no. 11, pp. 1293-1297, 2005.

[40] B. Seriolo, S. Paolino, A. Sulli, V. Ferretti, and M. Cutolo, "Bone metabolism changes during anti-TNF- $\alpha$ therapy in patients with active rheumatoid arthritis," Annals of the New York Academy of Sciences, vol. 1069, pp. 420-427, 2006. 


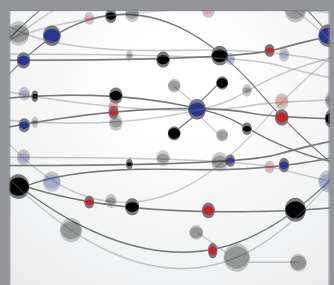

The Scientific World Journal
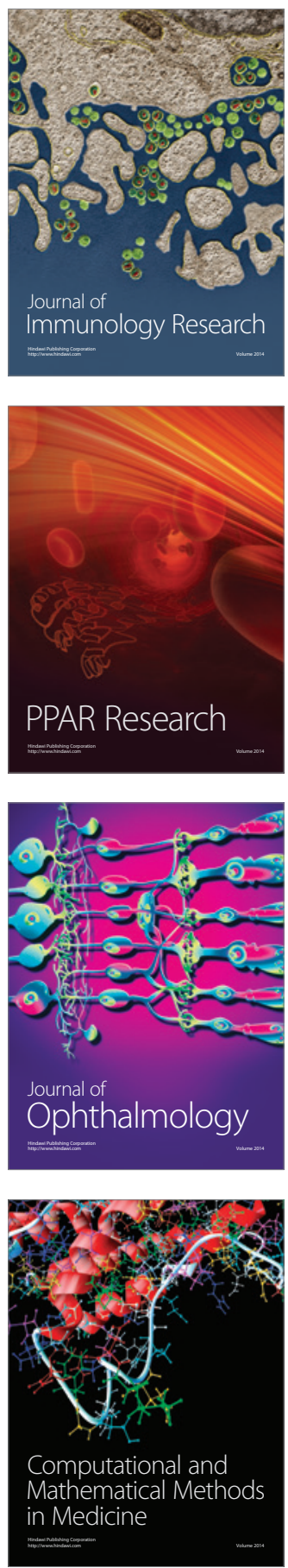

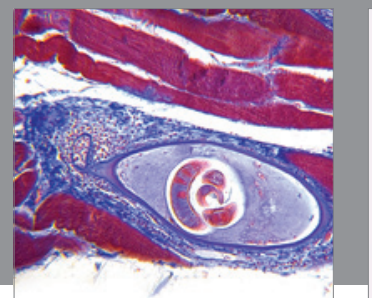

Gastroenterology

Research and Practice
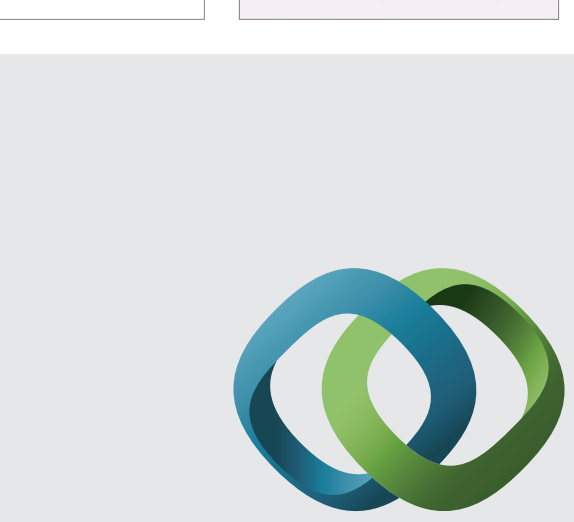

\section{Hindawi}

Submit your manuscripts at

http://www.hindawi.com
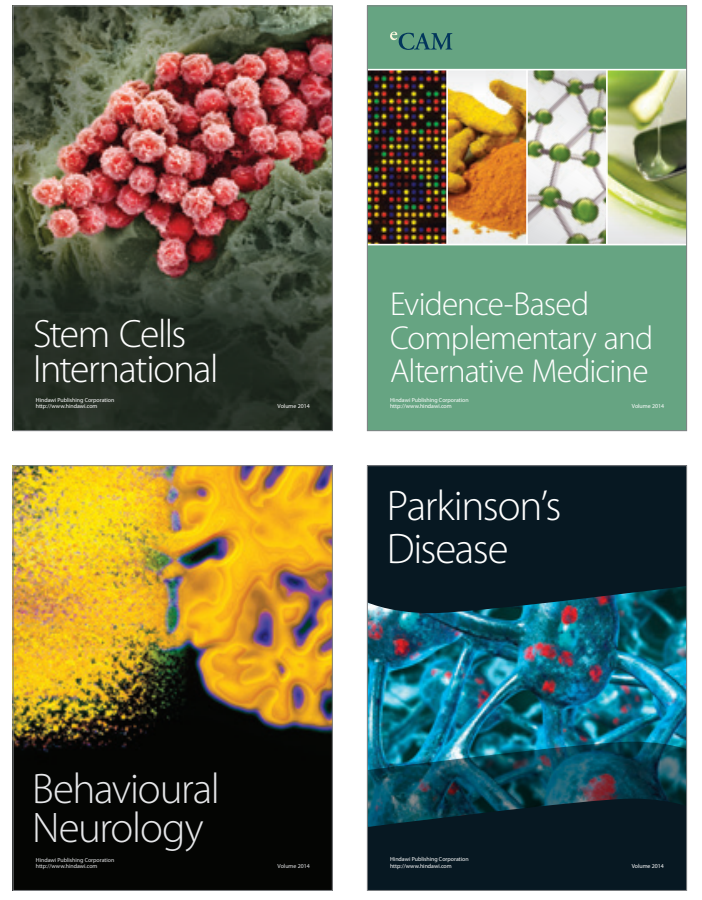
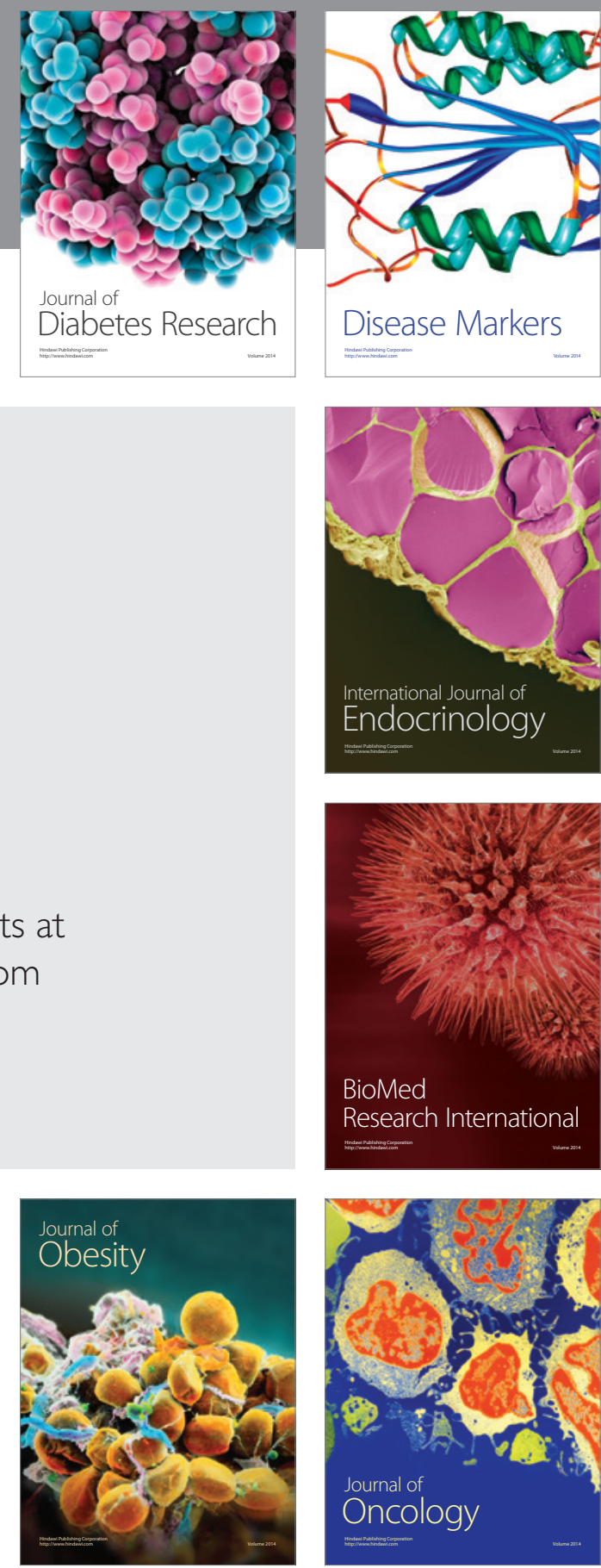

Disease Markers
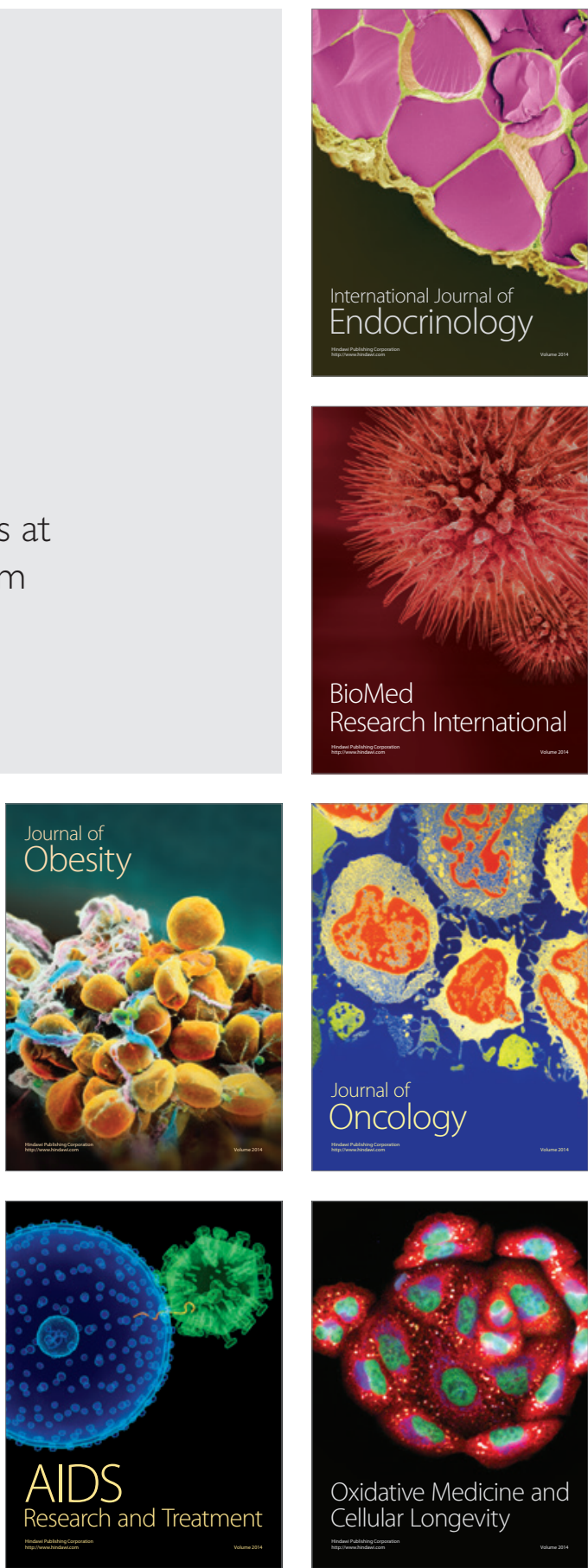